Check for updates

Cite this: RSC Adv., 2019, 9, 19720

Received 3rd May 2019

Accepted 14th June 2019

DOI: $10.1039 / c 9 r a 03305 j$

rsc.li/rsc-advances

\section{Green process to regenerate keratin from feathers with an aqueous deep eutectic solvent $\uparrow$}

\author{
Emmi-Maria Nuutinen, (D) *a Pia Willberg-Keyriläinen, ${ }^{a}$ Tommi Virtanen, ${ }^{a}$ \\ Alice Mija, $\mathbb{D}^{\mathrm{b}}{ }^{\text {Lauri Kuutti, }}{ }^{\mathrm{a}}$ Raija Lantto ${ }^{\mathrm{a}}$ and Anna-Stiina Jääskeläinen ${ }^{\mathrm{a}}$
}

Poultry feathers, a source of keratin, are a significant side stream from the food industry, for which valorization is essential considering the circular economy aspects. For this, ecofriendly processes are the tools that allow the easy and feasible transformation of the feathers. Deep eutectic solvents (DESs) are generally considered as inexpensive, relatively simple, mild and environmentally friendly solvents which can dissolve proteins from protein-rich biomasses. In this work, feathers were processed with an aqueous DES to produce a uniform keratin feedstock. The proposed DES is composed of non-toxic sodium acetate and urea, with a small amount of water. After the DES treatment, water was used to dilute the DES components and regenerate the dissolved keratin. The processing conditions were optimized in terms of keratin yield and properties by varying the dissolution time from $2 \mathrm{~h}$ to $24 \mathrm{~h}$ and temperature from $80{ }^{\circ} \mathrm{C}$ to $100{ }^{\circ} \mathrm{C}$. The yield of regenerated keratin was followed at different sodium acetate-urea molar ratios, and compared to the treatment performed with choline chloride-urea or $8 \mathrm{M}$ urea as reference solvents. Sodium acetate-urea in the molar ratio of $1: 2$ at $100{ }^{\circ} \mathrm{C}$ and with $6 \mathrm{~h}$ dissolution time dissolved $86 \%$ of the feathers with a regenerated keratin yield of $45 \%$. In the characterization of regenerated keratin, it was found that when the dissolution temperature was higher and the dissolution time longer, the disulfide and total sulfur content of feather keratin decreased, the range of molecular weights became wider, and some of the ordered secondary structure and crystallinity were lost.

\section{Introduction}

The poultry industry generates a vast amount of waste feathers annually. In 2018, it was estimated that broiler meat production alone was going to be approximately 92 million tons, ${ }^{1}$ with an estimated generation of 12 million tons of feathers worldwide. These feathers are usually disposed of in landfills, incinerated or recycled as a low-value animal feed. ${ }^{2}$ However, feathers consist of approximately $90 \%$ of a protein, keratin. As a renewable and biodegradable biopolymer, this keratin could also be applied in a more sustainable way in value added applications. However, this kind of utilization would require conversion of the complex feather structure into a more appropriate form such as micro- and nanoparticles, fibers, films, or hydrogels. ${ }^{2}$

${ }^{a}$ Solutions for Natural Resources and Environment, VTT, Technical Research Centre of Finland, Tietotie 2, FI-02044, Espoo, Finland. E-mail: emmi.nuutinen@vtt.fi; Tel: $+358406817126$

${ }^{b}$ Université Côte d'Azur, Université Nice-Sophia Antipolis, Institut de Chimie de Nice, UMR CNRS 7272, 06108 Nice Cedex 02, France

$\dagger$ Electronic supplementary information (ESI) available: Experimental procedure for preparation of the aqueous DES and other low melting mixtures, DSC and TGA for DESs, ATR-FTIR spectra and their deconvolution, NMR spectra and their deconvolution, DSC thermograms, and XRD patterns and their deconvolution. See DOI: 10.1039/c9ra03305j
Such non-toxic, biocompatible and biodegradable keratinous bioproducts could then find commercial applications in $e . g$. the food, cosmetics, agriculture, textile, composite and medical industries. $^{2}$

One attractive approach to obtain high quality keratin for material applications is to use specific solvents to extract the keratin from the feathers. Feather keratin is a fibrous structural protein $^{3}$ and generally feathers are known to be insoluble in common polar and non-polar solvents due to their extensive disulfide crosslinking, high content of hydrophobic residues ${ }^{4}$ and tight packing of ordered secondary structures, $\alpha$-helices and $\beta$-sheets, in polypeptide chains. ${ }^{5}$ Therefore, effective and profitable processes to extract the feather keratin are required. Usually, this extraction is done by disulfide cleavage which is achieved by reduction, oxidation, or sulfitolysis of the disulfide bonds. ${ }^{6}$ The chemicals which have the ability to ensure these reactions are often toxic, poorly recyclable and expensive to produce. Furthermore, the need for complex mixtures drives the need to develop more simple methods.

Ionic liquids (IL) are salts which are usually liquids at room temperature. ${ }^{7}$ ILs have attracted attention since they are considered as good solvents for inorganic and organic materials, they have an ability to be polar but non-coordinating solvents, they are immiscible with most organic solvents and 
they are non-volatile. ${ }^{7}$ In biomass fractionation, the dissolution with ILs is based on their capability to disrupt the hydrogen bonding within the component to be dissolved. ${ }^{8,9}$ ILs have also demonstrated their capacity in keratin extraction from wool ${ }^{\mathbf{1 0 , 1 1}}$ and feathers. ${ }^{12-17}$

Deep eutectic solvents (DESs) are a rather new type of solvents used in biomass processing. They were first presented by Abbott et al. ${ }^{18}$ as alternative solvents for ILs. DESs are composed of solid components which together form a mixture with a melting point lower than that of its individual components. ${ }^{8}$ The actual DES is obtained when the components are mixed in the specific molar ratio in which the system reaches its lowest melting point. ${ }^{19}$ However, mixing with other molar ratios may also produce mixtures with remarkably decreased melting temperatures. ${ }^{20}$ The drop in the melting point is due to the hydrogen bonding between the components which decreases the lattice energy of the system. ${ }^{18}$ Like ILs, DESs also usually have a low vapor pressure and are non-flammable. However, unlike ILs, DESs are commonly relatively easy and inexpensive to prepare ${ }^{19}$ and they are usually made of non-toxic, biodegradable and biocompatible components. ${ }^{20}$ Probably the most cited DES, choline chloride $(\mathrm{ChCl})$-urea in the molar ratio of $1: 2$, has a melting point of $12{ }^{\circ} \mathrm{C}$, notably lower than that of its individual components. ${ }^{21}$ DESs have also already demonstrated their potential in keratin extraction from wool. ${ }^{11,22-24}$

In this study, feather processing was investigated using a non-toxic and environmental friendly aqueous DES. The used DES was $90 \mathrm{wt} \%$ sodium acetate (NaOAc) - urea in the molar ratio of $1: 2$. This aqueous DES has shown great potential in protein extraction from Brewer's Spent Grain (BSG). ${ }^{20}$ Thermal characterization of the used DES was carried out using differential scanning calorimetry (DSC) and thermogravimetric analysis (TGA). In the feather processing, the effect of the dissolution time and temperature were studied using mass balances and different characterization methods for obtained regenerated keratin. Elemental analysis and direct colorimetric assay were used to study the disulfide, sulfhydryl and total sulfur contents, matrix-assisted laser desorption ionization time-of-flight mass spectrometry (MALDI-TOF MS) for the molecular weight range, attenuated total reflectance Fourier transform infrared spectroscopy (ATR-FTIR) and solid state nuclear magnetic resonance (NMR) for the secondary structures, X-ray powder diffraction (XRD) for the crystallinity and DSC for the thermal behavior.

\section{Experimental}

\subsection{Materials}

Sanitized chicken feathers supplied by Grupo Sada (Madrid, Spain) were ground into 2-15 $\mathrm{mm}$ pieces using an E-compactor (VTT, Finland) in which the feathers are pressed through a die using pan grinder rollers. Extra pure sodium acetate (NaOAc) was purchased from Honeywell (Germany), 99.0-100.6\% urea from Sigma-Aldrich (Germany) and 98\% choline chloride ( $\mathrm{ChCl})$ from Sigma-Aldrich (China).

\subsection{Differential scanning calorimetry (DSC) and thermogravimetric analysis (TGA)}

Preparation of the aqueous DES and other low melting mixtures is described in ESI. $\dagger$ A Mettler Toledo Differential Scanning Calorimeter (DSC820, Mettler Toledo GmbH, Switzerland) was used to perform the thermal behavior measurements. An approximately 5-15 $\mathrm{mg}$ sample was weighed into a pre-weighed $40 \mu \mathrm{l}$ aluminum crucible. The pans were closed by cold pressing and holes were punctured into the lids to release any pressure arising during heating. For analysis of the melting temperatures and enthalpies of the NaOAc-urea mixtures, the samples were

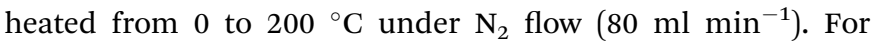
analysis of keratin, the samples were heated from -80 to $400{ }^{\circ} \mathrm{C}$ under $\mathrm{N}_{2}$ flow (80 $\mathrm{ml} \mathrm{min}^{-1}$ ). Heating and cooling rates of $10{ }^{\circ} \mathrm{C} \min ^{-1}$ were used in all cases. All experiments were performed in duplicate.

Thermogravimetric analysis (TGA) of the $1: 2$ NaOAc-urea mixtures were carried out using a Netzsch device (STA449F1, Netzsch Gerätebau GmbH, Germany) under $\mathrm{N}_{2}$ flow (40 $\mathrm{ml} \mathrm{min}^{-1}$ ). The analyzing temperature range was from 40 to $1000{ }^{\circ} \mathrm{C}$ with a heating rate of $5{ }^{\circ} \mathrm{C} \mathrm{min}^{-1}$.

\subsection{Elemental analysis}

Elemental analysis (C, H, N and S) was carried out using a FLASH 2000 Organic Elemental Analyzer (Thermo Scientific, Germany). Before the analysis, samples were dried overnight in an oven at $105{ }^{\circ} \mathrm{C}$ in order to remove excess moisture. The average values of two duplicate samples were measured and reported.

\subsection{Protein content}

Total protein content can be approximated by multiplying the nitrogen content by a factor corresponding to the average nitrogen content of proteins. The nitrogen content of the native feathers was determined by elemental analysis and the protein content was then determined using 6.66 as a conversion factor. ${ }^{25}$ The soluble protein content was determined with a twostep method. First, diluted aqueous samples were precipitated using the commercial Compat-Able ${ }^{\mathrm{TM}}$ Protein Assay Preparation Reagent Set (Thermo Scientific, USA). ${ }^{26}$ Then the protein content was measured using the commercial BCA protein assay kit (Thermo Scientific, USA). ${ }^{27}$

\subsection{Sulfur compounds}

The direct colorimetric solid phase assay for free sulfhydryl groups and disulfide bonds described by Chan and Wasserman ${ }^{28}$ was used to determine the sulfhydryl (SH) and disulfide (S-S) contents in the native feathers and in the regenerated keratin samples. Water was used to dilute samples when needed. 2-Nitro-5thiosulfobenzoate $\left(\mathrm{NTSB}^{2-}\right)$ needed in the assay was synthesized according to Thannhauser et $a .^{29}$ The concentrations were calculated using $13600 \mathrm{M}^{-1} \mathrm{~cm}^{-1}$ as an extinction coefficient and total sulfhydryl content was assumed to be given by the sum of $[\mathrm{SH}]+2$ [S-S]. Between 2-4 replicates were measured and the average value of each sample was reported. 
2.6 Matrix-assisted laser desorption ionization time-of-flight mass spectrometry (MALDI-TOF MS)

Matrix-assisted laser desorption ionization time-of-flight mass spectrometry (MALDI-TOF MS) was applied to determine the ranges of molecular weights of the keratin samples. Before the measurement, keratin samples were dissolved in a mixture of $1.5 \%$ dithiothreitol (DTT), $0.5 \mathrm{M}$ tris hydrochloride $(\mathrm{HCl}), 10 \%$ glycerol and $2 \%$ sodium dodecyl sulfate (SDS). Sinapinic acid was selected as the matrix and dissolved to saturation in a mixture of $0.1-0.3 \%$ trifluoroacetic acid (TFA) and $50 \%$ acetonitrile. One microliter of the matrix mixture and sample were placed on the target plate and dried under air. The analysis was conducted using a Bruker mass spectrometer Autoflex II Maldi-TOF LRF50-CID (Bruker Daltonik GmbH, Germany).

\subsection{Fourier transform infrared spectroscopy (FTIR)}

A Fourier transform infrared spectroscopy (FTIR) spectrometer equipped with an attenuated total reflectance (ATR) diamond crystal (Nicolet iS50, Thermo Scientific, USA) was used for the structural studies. All spectra were collected using 32 scans in a spectral range of $4000-400 \mathrm{~cm}^{-1}$ and with a spectral resolution of $4 \mathrm{~cm}^{-1}$. Several spectra were collected from different locations of each sample, and the average of these spectra was used in the analysis. The spectra were processed with OriginPro 2017 software. Deconvolution of the bands was carried out using OriginPro 2017 software (Multiple Peak Fit) and Gaussian fit following the studies of Tsuboi et al. ${ }^{30}$ and Rintoul et al., ${ }^{31}$ and the bands for each secondary structure were assigned according to the studies of Barth $^{32}$ and Miyazawa \& Blout. $^{33}$ Deconvolutions were carried out for three or four spectra of each sample, and the average and standard deviation were reported.

\subsection{Nuclear magnetic resonance spectroscopy (NMR)}

The ${ }^{13} \mathrm{C}$ cross polarization (CP) magic angle spinning (MAS) Nuclear magnetic resonance spectroscopy (NMR) measurements were performed using an Agilent DD2 600 spectrometer (Agilent Technologies, USA), with magnetic flux density of 14.1 T and equipped with a $3.2 \mathrm{~mm}$ T3 MAS NMR probe operating in double resonance mode. The MAS rate in experiments was 10 $\mathrm{kHz}$, the number of scans was 10000 with a $6.0 \mathrm{~s}$ delay between successive scans, and CP contact time was $1.3 \mathrm{~ms}$. The spectra were processed using TopSpin 3.5 and OriginPro 2017 software. Deconvolution of the peak was carried out using OriginPro 2017 software (Multiple Peak Fit) and Gaussian functions. Deconvolution was performed based on the studies of Duer et al. ${ }^{34}$ and Idris et al. ${ }^{12}$

\subsection{X-ray powder diffraction (XRD)}

X-ray powder diffraction (XRD) was used to determine the semicrystallinity of feather keratin samples. A Philips X'pert Pro diffractometer (PW 1130/00, Philips, The Netherlands) was used with Ni-filtered $\mathrm{Cu} \mathrm{K} \alpha$ radiation $(\lambda=1.541 \AA)$. The data were obtained with geometry in which the sample was kept in place and the tube and counter were in motion. The X-ray beam was generated at $45 \mathrm{kV}$ and $30 \mathrm{~mA}$. Diffraction intensities were recorded with $2 \theta$ ranging from $4^{\circ}$ to $60^{\circ}$ at a step size of $0.079^{\circ}$ and a scan step time of $390 \mathrm{~s}$. The data was processed using X'Pert and OriginPro 2017 software. Deconvolution of the peaks was carried out using OriginPro 2017 software (Multiple Peak Fit) and Gaussian functions. Deconvolution was performed based on the study of Cao \& Billows. ${ }^{35}$

\subsection{Dissolution and regeneration of feather keratin}

Regenerated keratin samples were obtained by dissolving the feathers in the aqueous DES after which the dissolved keratin was regenerated in water. $3 \mathrm{~g}$ of ground feathers were added in $147 \mathrm{~g}$ of preheated $\left(80-100{ }^{\circ} \mathrm{C}\right)$, clear, freshly prepared solvent. The used solvents were $90 \mathrm{wt} \%$ NaOAc-urea in the molar ratio of $1: 2$ and $1: 3.90 \mathrm{wt} \% \mathrm{ChCl}-$ urea in the molar ratio of $1: 2$ and $8 \mathrm{M}$ urea were used as reference solvents for NaOAc-urea. The solution was mixed from $2 \mathrm{~h}$ to $24 \mathrm{~h}$. After the desired dissolution time, the solution was filtered through a metallic wire mesh $(90 \mu \mathrm{m})$ in a heated $\left(105{ }^{\circ} \mathrm{C}\right)$ and pressurized $(3-5$ bar) filtration unit. The solid residue was washed with Milli-Q water and freeze-dried. The filtrate was added to $350 \mathrm{ml}$ of Milli-Q water to regenerate the dissolved feather keratin and dissolve the DES components. Regenerated keratin was then filtered in a Büchner funnel $(60 \mu \mathrm{m})$, washed with Milli-Q water and freeze-dried.

\section{Results and discussion}

\subsection{Preparation and characterization of the aqueous DES and low melting mixtures}

In this study, urea was coupled with NaOAc to form a mixture which had a lower melting point than urea and NaOAc alone. The DSC results (Table S1 and Fig. S1 $\dagger$ ) indicate that the lowest melting temperature $\left(85{ }^{\circ} \mathrm{C}\right)$ was achieved for the mixture of $\mathrm{NaOAc}$ and urea in the molar ratio of $1: 2$. Based on this result, it was decided to use this ratio as DES in this study. In earlier studies, NaOAc trihydrate-urea mixtures are reported to reach their eutectic point $\left(30{ }^{\circ} \mathrm{C}\right.$ and $\left.33{ }^{\circ} \mathrm{C}\right)$ in the molar ratio of $2: 3 .^{36,37}$ However, in these studies, it should be noted that the portion of water changes along with the portion of NaOAc trihydrate, and in DES systems, water decreases the melting point. This is due to the capability of water to decrease the lattice energy of the mixture through new hydrogen bonds. ${ }^{19}$ To speed up the melting and decrease the viscosity of the solution without completely destroying the hydrogen bonding between the DES components, $10 \mathrm{wt} \%$ of water was added to the $1: 2$ NaOAc-urea mixture. In this way the melting temperature decreased from $85{ }^{\circ} \mathrm{C}$ to $38{ }^{\circ} \mathrm{C}$ (Table S1 $\dagger$ ). TGA was carried out to identify the suitable processing temperature of the chosen mixture. Because the loss of water can be controlled with the cooling system in the process, it is suggested that the processing temperature of this aqueous DES should be between $38{ }^{\circ} \mathrm{C}$ and $105{ }^{\circ} \mathrm{C}$ in order to avoid the decomposition of DES components (Fig. S2†). 


\subsection{Dissolution and regeneration of feather keratin}

When the feathers were processed with the aqueous DES, three different fractions were obtained: undissolved feathers, regenerated keratin, and soluble keratin (Fig. 1). After the separation of the undissolved feathers with heated and pressurized filtration system $(90 \mu \mathrm{m})$, the dissolved keratin regenerated into water. Moreover, not all of the dissolved keratin precipitated in the regeneration step, but rather dissolved in water together with NaOAc and urea. This fraction is called soluble keratin. The success of the processing was studied using the mass balances. The gravimetric yields of undissolved feathers and regenerated keratin were obtained by weighing the freeze-dried samples. The soluble keratin content was determined with a BCA protein assay kit. In this study, an unidentified part is also reported. This fraction corresponds to the part of the applied feathers $(3 \mathrm{~g})$ which was not captured when mass balances were calculated.

The effect of the dissolution time in the feather processing was investigated using a dissolution temperature of $90{ }^{\circ} \mathrm{C}$ and varying the time from $2 \mathrm{~h}$ to $24 \mathrm{~h}$. The time series at $90{ }^{\circ} \mathrm{C}$ was performed in duplicate to illustrate the standard error. The results (Fig. 2a) show that when the dissolution time increased from $2 \mathrm{~h}$ to $14 \mathrm{~h}$, the yield of regenerated keratin increased from $1 \%$ to $35 \%$ and that of soluble keratin from $10 \%$ to $21 \%$, while the undissolved feather fraction decreased from $77 \%$ to $20 \%$. When the dissolution time was further increased to $16 \mathrm{~h}$ and $24 \mathrm{~h}$, the yield of undissolved feather fraction continued to decrease down to $11 \%$, and regenerated and soluble keratin also started to decrease. On the other hand, the unidentified fraction increased to $38 \%$. Based on these results, it appears that in feather processing using the aqueous DES, the dissolution of feathers as well as the generation of regenerated and soluble keratin occurred concurrently. However, when a certain level of regenerated keratin was reached, the growth of soluble and unidentified fractions appeared to be the main phenomenon. This could indicate the cleavage of protein chains into low molecular weight components such as polypeptides or amino acids.

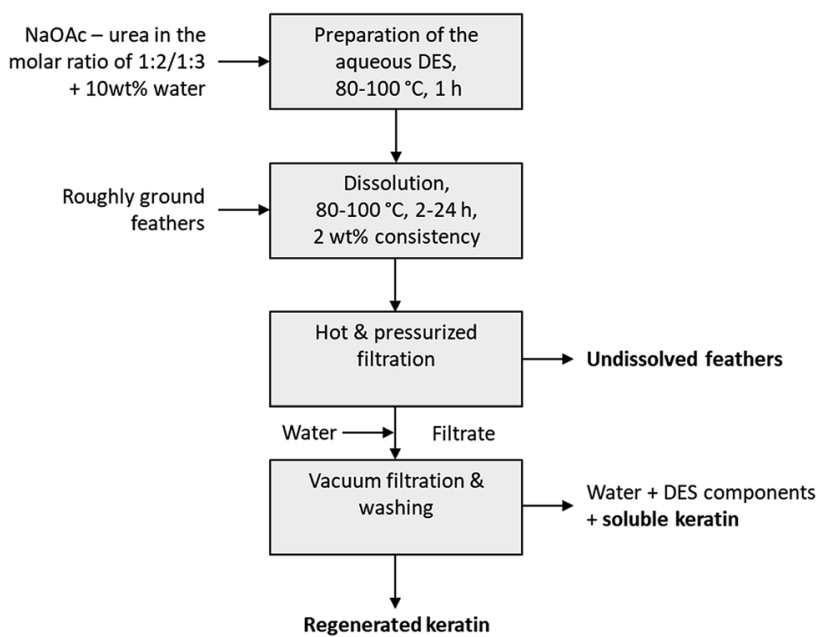

Fig. 1 Operational procedure for feather treatment with an aqueous DES.
The effect of the dissolution temperature was investigated using $6 \mathrm{~h}$ dissolution time and varying the dissolution temperature from $80{ }^{\circ} \mathrm{C}$ to $100^{\circ} \mathrm{C}$. Fig. $2 \mathrm{~b}$ represents the results. It can be detected that when the temperature was increased from $80{ }^{\circ} \mathrm{C}$ to $100{ }^{\circ} \mathrm{C}$, the yield of regenerated keratin increased from $0 \%$ to $45 \%$ and that of soluble keratin from $7 \%$ to $21 \%$ whereas the fraction of undissolved feathers decreased from $83 \%$ to $14 \%$. It is clear that temperature has an important effect in the dissolution. It is suggested that at higher temperature the dissolution is more effective due to lower viscosity of the solvent and higher mobility of the solvent components compared to the lower temperatures. ${ }^{38}$ When the viscosity of the system decreases, the mass transfer and diffusion of DES components into the structures are enhanced. ${ }^{23,39}$ The keratin yields obtained at $100{ }^{\circ} \mathrm{C}$ and with $6 \mathrm{~h}$ dissolution time are comparable to some other feather processing methods. In this case, $86 \%$ of feathers were dissolved in the aqueous DES. In previous studies, Sharma et al. ${ }^{\mathbf{4 0}}$ and Yin et $a{ }^{4 \mathbf{4 1}}$ have obtained keratin yields of $80 \%$ and $90 \%$ for acid precipitated keratin, respectively. Sharma et $a l .{ }^{40}$ dissolved feathers in $0.5 \mathrm{M}$ sodium sulfide while in the study of Yin et $a .^{41}$ feathers were processed with Shindai method in which feathers are treated with ethanol pretreatment, hydrochloric acid pretreatment and 2-mercaptoethanol.

In protein denaturation, the role of urea as a denaturant is well established. It has also been used in feather keratin extraction with other chemicals such as reducing agents. ${ }^{\mathbf{4 2 - 4 4}}$ It is known that urea has an ability to unfold proteins. ${ }^{45}$ As a reference to the aqueous DES, the effect of $8 \mathrm{M}$ urea was studied to dissolve feathers at $90^{\circ}$ for $6 \mathrm{~h}$. As expected, the results (Fig. 2c) show that $8 \mathrm{M}$ urea was also able to dissolve 55\% of the feathers. However, with urea, none of the desired fraction, regenerated keratin, was observed. The difference is visualized in Fig. 3. With $8 \mathrm{M}$ urea, keratin did not start to precipitate before the $\mathrm{pH}$ of the solution was adjusted to 4.6. This kind of additional step could further complicate the recycling of the solvent. It is clear that the behavior of urea in DES differs from its behavior in aqueous solutions due to the strong interactions between the DES components. The effect of urea in the NaOAc-urea mixture was also studied by increasing to portion of urea in a way that the molar ratio of the aqueous NaOAc-urea mixture was $1: 3$. It was found that this molar ratio was able to dissolve $80 \%$ of the feathers with the formation of $30 \%$ of regenerated keratin at $90{ }^{\circ} \mathrm{C}$ for $6 \mathrm{~h}$ which was more than with the molar ratio of $1: 2$ or $8 \mathrm{M}$ urea (Fig. $2 \mathrm{c}$ ). Thus, the yields of different fractions can be tailored not only by tuning the dissolution conditions but also the molar ratio of NaOAc-urea mixture.

The other main component in the aqueous DES is composed of sodium cation and acetate anion ( $\mathrm{NaOAc})$. It is suggested that in some DES systems, salts may act as stabilizing agents for proteins as they increase the ionic strength of the environment. ${ }^{46}$ It has also been suggested that the protein can remain stable in some DES systems because the hydrogen bonding between urea and anion limits the penetration of urea molecules deep into the protein core, and DES components preferably interact with the surface residues of protein. ${ }^{47}$ 
(a)

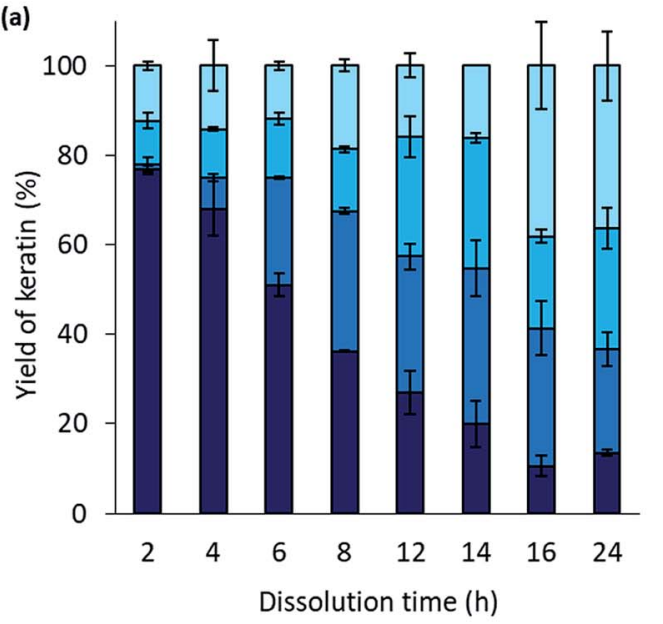

(b)

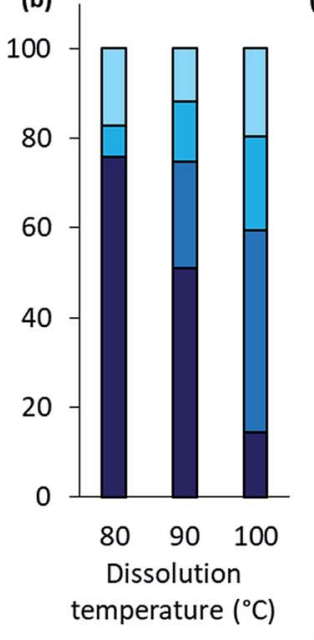

(c)

Fig. 2 The yields of feather keratin in each fraction using 90 wt\% $1: 2 \mathrm{NaOAc}-$ urea (a) at $90^{\circ} \mathrm{C}$ for $2-24 \mathrm{~h}$, (b) at $80-100{ }^{\circ} \mathrm{C}$ for $6 \mathrm{~h}$, and (c) using $8 \mathrm{M}$ urea, $90 \mathrm{wt} \% 1: 3 \mathrm{NaOAC}-$ urea and $90 \mathrm{wt} \% 1: 2 \mathrm{ChCl}$-urea at $90{ }^{\circ} \mathrm{C}$ for 6 .

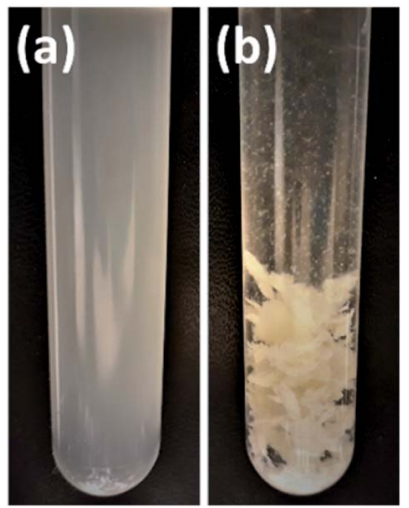

Fig. 3 Samples after processing with (a) 8 M urea and (b) aqueous DES ( $1: 2 \mathrm{NaOAc}$-urea) when water was added to the solution to regenerate the keratin.

When $90 \mathrm{wt} \%$ DES consisting of $\mathrm{ChCl}$ and urea in the molar ration of $1: 2$ was tested for the feather dissolution at $90{ }^{\circ} \mathrm{C}$ for $6 \mathrm{~h}$, no regenerated keratin was formed and the undissolved fraction was $87 \%$ (Fig. 2c). In addition to urea, the role of anion is suggested to be important in the dissolution processes with DESs. ${ }^{22}$ Moore et al. ${ }^{22}$ suggested that wool dissolution in $\mathrm{ChCl}-$ urea is due to the capability of highly polar anion and urea to disturb the hydrogen bonding, the disulfide and electrostatic bonds present in the wool. It has been suggested that because the acetate anion has higher basicity than chloride, it could more effectively disturb the hydrogen bonding within the material. ${ }^{48}$ The different cation may also partly explain the difference between the aqueous DESs. However, the effect of cations is suggested to be less important than that of anions. ${ }^{22}$

A small addition of water is also proposed to have a positive effect on the dissolution with NaOAc-urea DES, as it decreases the viscosity of the mixture, thus facilitating the mass transfer and in this way the dissolution of the system..$^{20}$ However, it should be taken into account that too much water can be harmful because it starts to compete with the hydrogen bonding which is responsible for the dissolution. ${ }^{9}$

\subsection{Characterization of regenerated keratin}

3.3.1 Sulfur compounds. Feather keratin possesses disulfide bonds that restrict its dissolution. In feather keratin, sulfur is located in disulfide bonds $(\mathrm{S}-\mathrm{S})$, in sulfhydryl groups of cysteine or in methionine. ${ }^{4}$ Because the content of methionine is considered to be low, ${ }^{4}$ the sulfur is almost completely located in disulfide bonds and sulfhydryl groups. In order to understand the dissolution with aqueous DES, the disulfide, free sulfhydryl and total sulfur contents were studied with the direct colorimetric assay and elemental analysis. The results (Fig. 4) indicate that the DES treatment decreased the concentration of disulfide bonds from $68.5 \mathrm{nmol} \mathrm{mg}^{-1}$ to $24.5 \mathrm{nmol} \mathrm{mg}^{-1}$ when the dissolution time was increased from $0 \mathrm{~h}$ to $16 \mathrm{~h}$. A similar trend has been observed in other studies in which feathers were treated by steam explosion. ${ }^{\mathbf{4 9 , 5 0}}$

After the cleavage of one disulfide bond, two cysteine residues are formed. Between the regenerated keratin samples, an increasing trend of sulfhydryl groups could be observed towards the longer dissolution time as the concentration increased from $1.3 \mathrm{nmol} \mathrm{mg}^{-1}$ to $3.0 \mathrm{nmol} \mathrm{mg}{ }^{-1}$ between the $2 \mathrm{~h}$ and $16 \mathrm{~h}$ dissolution times (Fig. 4a). However, the free sulfhydryl group contents in regenerated keratin samples were lower than in the native feathers $\left(11.7 \mathrm{nmol} \mathrm{mg}^{-1}\right)$. The decrease in the sulfhydryl content indicates the destruction of cysteine residues and the further reactions of the free sulfhydryl groups into sulfurcontaining volatiles during the processing: ${ }^{49}$ The loss of sulfur can also be seen in Fig. 4b, in which total sulfur and sulfhydryl contents measured by elemental analysis and colorimetric assay are presented. Both analyses show a similar decreasing trend in sulfur content. However, a difference can be seen in the percentages. The difference between the methods can probably 

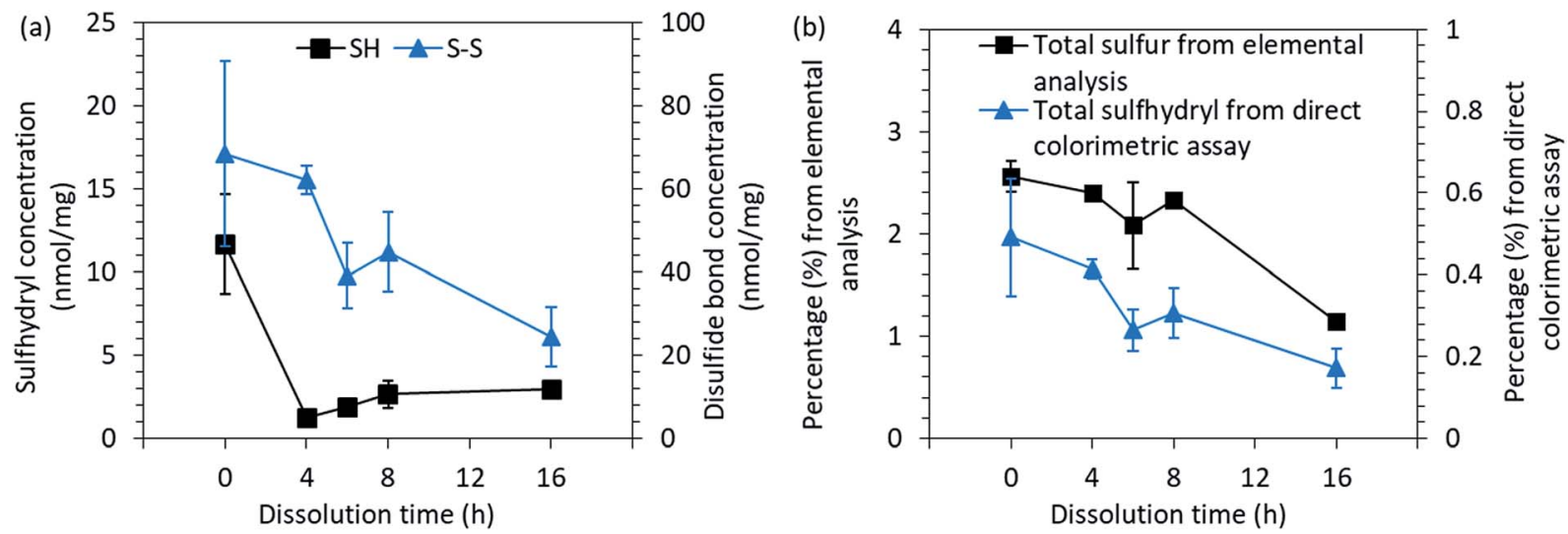

Fig. 4 (a) The concentration of free sulfhydryl groups $(\mathrm{SH})$ and disulfide bonds $(\mathrm{S}-\mathrm{S})$ and $(\mathrm{b})$ the total sulfur and sulfhydryl contents present in the native feathers and in regenerated keratin.

be explained by the accessibility to the structure. In elemental analysis, the structure of the sample was destroyed and all the sulfur could be detected, whereas in the colorimetric assay, the color reagents may not have been able to react with all the sulfhydryl groups because not all the keratin was dissolved in the reaction buffer. However, although the colorimetric assay may not give the absolute values, a common trend could be discerned.

3.3.2 Molecular weight. MALDI-TOF MS was used to study changes in the molecular weights of the regenerated keratin samples. In the literature, it is proposed that the molecular weights of feather keratin is around $10 \mathrm{kDa}^{.51}$ The native feathers could not be dissolved completely and therefore no reliable MALDI-TOF spectra could be measured. Fig. 5, which represents the data obtained from MALDI-TOF MS, shows that with longer dissolution time (Fig. 5a-c) and with higher dissolution temperatures (Fig. 5d), the range of molecular weights became wider. After $4 \mathrm{~h}$ dissolution time, a wide peak can be observed around $10000 \mathrm{~m} / \mathrm{z}$ and a smaller wide peak at approximately $5000 \mathrm{~m} / \mathrm{z}$. When the dissolution time and temperature increased, wide peaks started to appear at approximately $7000 \mathrm{~m} / \mathrm{z}$ and $8000 \mathrm{~m} / \mathrm{z}$, and peak at $10000 \mathrm{~m} / \mathrm{z}$ started to decrease. This could indicate degradation of the keratin chains. This supports the conclusion that the increase in the soluble keratin yield and the loss in total yield were due to degradation of the polypeptide backbones.

3.3.3 Secondary structures. Feather keratin presents two different ordered conformations in its secondary structure. The polypeptide chain can either curl into $\alpha$-helices or bond into plated sheets, $\beta$-sheets. In addition to these ordered structures, feather keratin includes disordered structures, random coil, and chain reversal regions, $\beta$-turns, between the $\beta$-sheets. ${ }^{52}$

ATR-FTIR was used to study the chemical structure and more specifically the secondary structures of the feather keratin. Although MALDI-TOF MS indicated that the keratin molecules started to degrade during the DES processing, the FTIR spectra (Fig. S3†) suggest that most of the polypeptide backbone in the regenerated keratin was retained. This can be observed from the shape of the spectra and especially from the amide I and II bands. The amide I band at $1600-1700 \mathrm{~cm}^{-1}$ is mainly assigned to the $\mathrm{C}=\mathrm{O}$ stretching vibration, while the amide II band at $1480-1570 \mathrm{~cm}^{-1}$ originates from out-of-phase combination of $\mathrm{NH}$ bending and $\mathrm{CH}$ stretching vibration. ${ }^{32}$ The amide I-II vibrations are known to be less affected by the nature of the side chains but rather by the conformation of the polypeptide backbone..$^{32}$ Therefore, these bands could also be used to study the secondary structures of proteins. In order to investigate the secondary structures more closely, the overlapping bands present in the amide I-II bands were determined by the deconvolution of these bands in their individual components

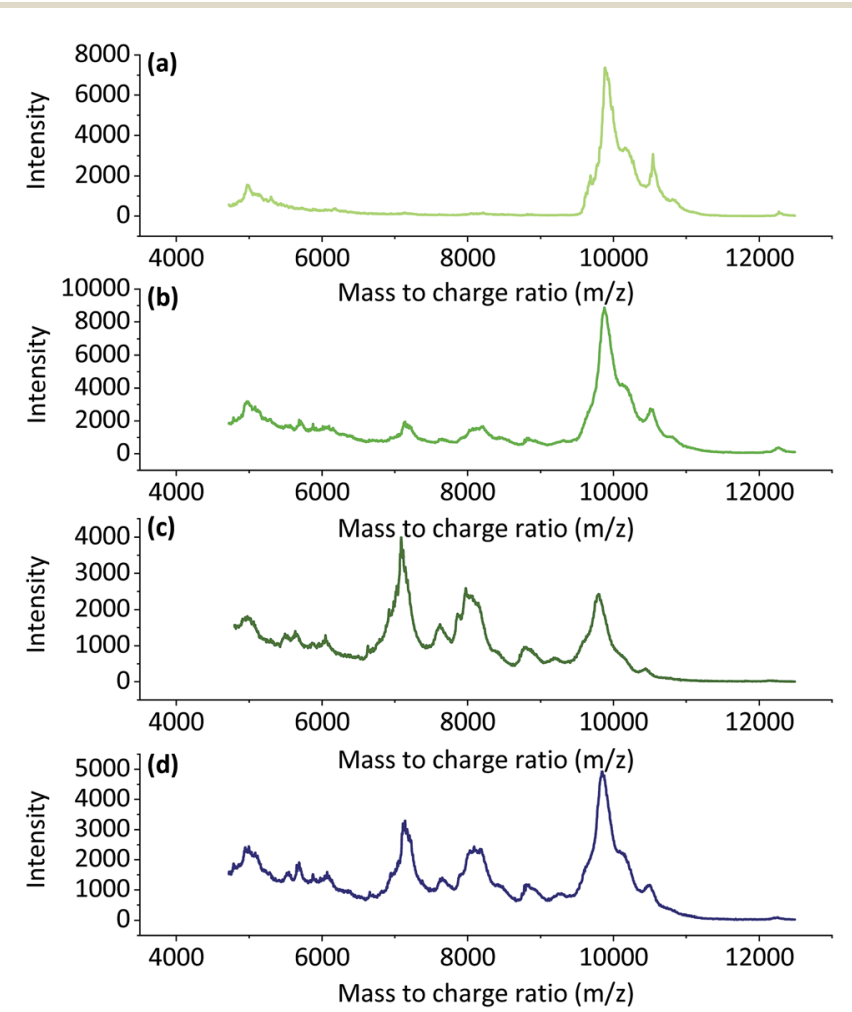

Fig. 5 The MALDI-TOF spectra for regenerated keratin at $90^{\circ} \mathrm{C}$ for (a) $4 \mathrm{~h}$, (b) $8 \mathrm{~h}$, (c) $16 \mathrm{~h}$ and (d) at $100{ }^{\circ} \mathrm{C}$ for $6 \mathrm{~h}$. 
(Fig. S4 $\dagger$ ). The results obtained from band fitting are summarized in Fig. 6a. The data obtained from the deconvolution shows that when the dissolution time was increased from $0 \mathrm{~h}$ to $16 \mathrm{~h}$, the disordered structure, random coil, increased from $55.4 \%$ to $71.6 \%$ and the portion of $\alpha$-helix \& random coil increased from $2.6 \%$ to $3.9 \%$, while the percentage of $\beta$-sheet and turns decreased from $30.5 \%$ and $11.5 \%$ to $19.7 \%$ and $4.8 \%$, respectively. The data and results obtained from the deconvolution should be treated with caution, as the parameter choice is rather subjective. However, although the values obtained should not be interpreted as absolute, a common trend can be concluded.

In addition to the ATR-FTIR, NMR was also used to investigate the molecular conformations and dynamics in the feather keratin. The spectra obtained (Fig. S5†) are characteristics for the feather keratin, ${ }^{\mathbf{1 2}}$ and are similar to each other with slight differences in the intensities, especially in the alkyl and $\alpha$ carbon region (10-70 ppm). This indicates that no major changes in the protein backbone, but rather some changes in the conformation and side chains, occurred during the processing. ${ }^{34}$ The peak at $176 \mathrm{ppm}$ is assigned to carbonyl carbons, and these carbons give slightly different shifts depending on whether they are located in $\alpha$-helix or $\beta$-sheet. ${ }^{34}$ Usually, $\alpha$-helix gives a shift at higher ppm than $\beta$-sheet. ${ }^{34}$ To detect the overlapping peaks and different secondary structures in the peaks in the 176 ppm region, deconvolution was carried out (Fig. S6†). The results are summarized in Fig. 6b. When the native feathers are compared to the regenerated keratin sample which was treated at $90{ }^{\circ} \mathrm{C}$ for $4 \mathrm{~h}$, the percentage of $\beta$-sheet and random coil increased from $84.6 \%$ to $90.4 \%$, while $\alpha$-helix decreased from $15.4 \%$ to $9.6 \%$. With longer dissolution time, the portion of $\beta$-sheet and random coil started to decrease and $\alpha$-helix to increase, and after $16 \mathrm{~h}$ the percentage of $\beta$-sheet and random coil was $86.2 \%$ and that of $\alpha$-helix was $13.8 \%$. The discussion of the comparison between the results obtained from NMR and ATR-FTIR is difficult as NMR gives the percentage of $\beta$-sheet and random coil together and $\alpha$-helix separately while in ATR-FTIR the percentages of $\beta$-sheet and random coil are given separately and $\alpha$-helix and random coil together. However, based on both methods, it seems that some of the ordered secondary structure was lost during the processing.

The changes of secondary structures was also noticed in DSC studies (Fig. S7 $\dagger$ ). In the native feathers the endothermic peak at $233{ }^{\circ} \mathrm{C}$ indicates the melting of $\alpha$-helices. ${ }^{53,54}$ For regenerated keratin samples this peak appeared already at $223{ }^{\circ} \mathrm{C}$ and is broadened compared to the native feathers. The shift towards lower temperatures and the change in the shape may indicate a greater portion of disordered structure ${ }^{12}$ or higher moisture content $^{53}$ in the regenerated keratin compared to the native feathers.

3.3.4 Crystallinity. As well as the disulfide bonding, the crystallinity is also believed to be an important factor in keratin's strength and stiffness. ${ }^{50}$ XRD was used to study and compare the crystallinity of the native feathers and regenerated keratin samples. Fig. $\mathrm{S} 8 \dagger$ represents the XRD patterns obtained. The patterns clearly indicate that all the samples were semi-crystalline keratin, with most notable peaks at approximately $9^{\circ}$ and $20^{\circ}$. In the native feathers, the peak at $9^{\circ}$ is broader than in regenerated keratin samples and starts already at approximately $4^{\circ}$. Furthermore, a small and broad peak at $40^{\circ}$ can be observed. On the other hand, in the processed keratin samples, new small sharp peaks can be observed. Different spacing as well as peak positions in XRD patterns indicate different structures and arrangements of crystals. ${ }^{55}$ In regenerated keratin samples, these new peaks most probably indicate new crystalline structures which appeared during regeneration. The most significant peaks at $9^{\circ}$ and $20^{\circ}$ correspond to the crystalline spacing of $9.8 \AA$ and
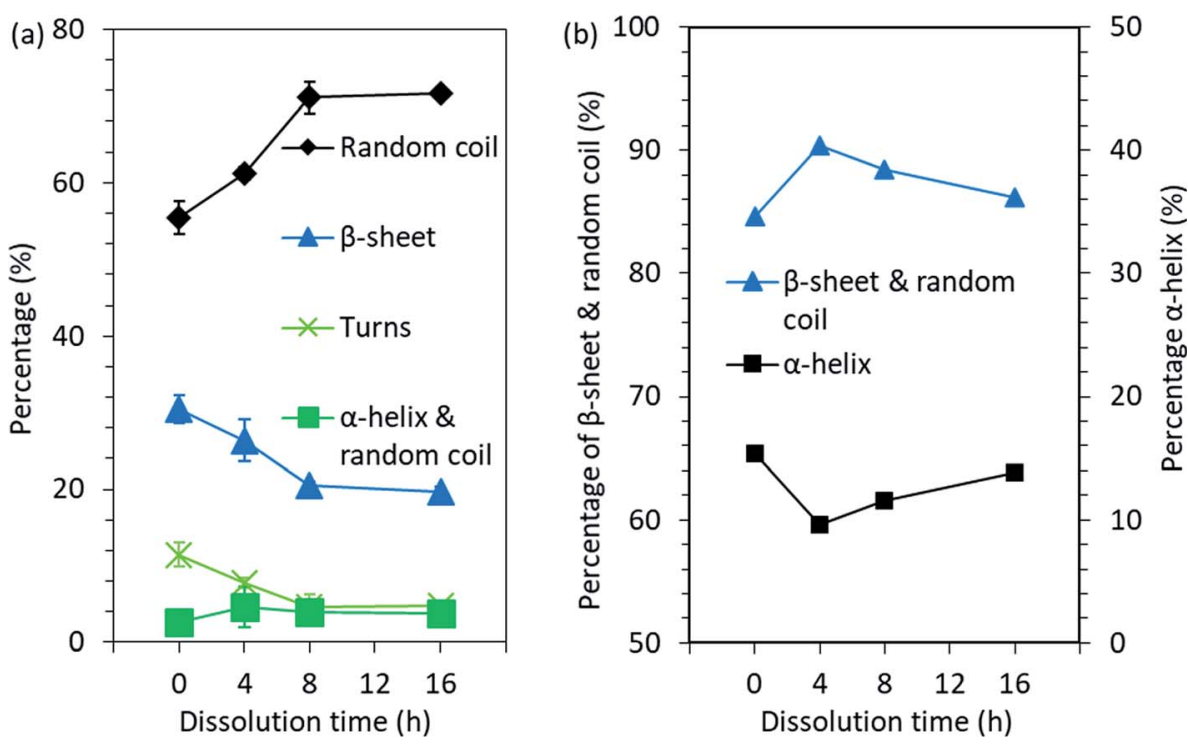

Fig. 6 Changes in the secondary structure of feather keratin after the processing obtained from (a) the FTIR spectra band fitting and (b) the NMR spectra peak fitting. 


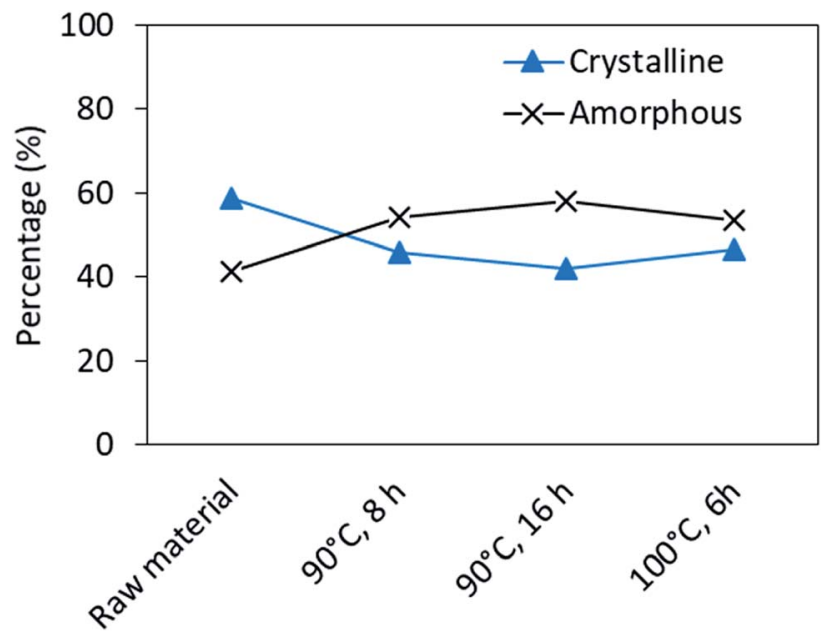

Fig. 7 Changes in the crystallinity of regenerated keratin after processing, obtained from the deconvolution of XRD patterns.

$4.4 \AA$, respectively. ${ }^{55}$ In all samples, the peaks at $9^{\circ}$ have higher intensity than those at $20^{\circ}$, and this difference is greatest in the native feathers. The diffraction spot at $5.1 \AA$ $\left(2 \theta=17.8^{\circ}\right)$ is associated with $\alpha$-helix, the spot at $4.65 \AA$ ( $2 \theta=$ $\left.19^{\circ}\right)$ with $\beta$-sheet, and the spot at $9.8 \AA\left(2 \theta=9^{\circ}\right)$ with both $\alpha$ helix and $\beta$-sheet. ${ }^{35,56}$ Due to the tight overlapping of $\alpha$-helix and $\beta$-sheet, their unambiguous assignment to individual components is not possible. However, deconvolution of crystalline and amorphous intensity profiles could be carried out in order to determine the portion of crystallinity in the samples (Fig. S9†). ${ }^{35}$ The results (Fig. 7) indicate that the degree of crystallinity decreased from $58.5 \%$ to $42.0 \%$ after $16 \mathrm{~h}$ of DES processing. The loss of the crystalline and ordered secondary structures is most probably related to the cleavage of disulfide bonds and polypeptide chains as well as to the change of interactions like hydrogen bonding present in feather keratin.

\section{Conclusions}

In the present study, the processing of waste feathers using an aqueous, inexpensive and food-grade DES was successfully demonstrated. It is suggested that the processing of feathers with aqueous DES is a complex system in which urea, sodium acetate as well as water take part. Regenerated keratin, which could be further applied in biomaterials, was obtained by precipitating the dissolved feather in water. In the characterization process of the regenerated keratin, it was observed that no major chemical changes occurred in the polypeptide backbone, and the part which precipitated and regenerated into water had the ability to either retain or rearrange part of its ordered and crystalline structure. The dissolution of keratin and the structural differences of regenerated keratin are proposed to be due to the ability of the aqueous DES to disturb the interactions within the feather keratin, cleave the disulfide bonds and partly break down the polypeptide backbone of keratin. However, although some structural differences took place after the aqueous DES processing, this method is proposed to represent a rather gentle and, with further optimization, potential processing method in which the properties as well as the yields of different fractions can be tailored by tuning the dissolution conditions and molar ratio of DES.

\section{Conflicts of interest}

There are no conflicts to declare.

\section{Acknowledgements}

Funding for this work was received from the European Union's Horizon 2020 Research and Innovation program under Grant Agreement 723268 on a project KaRMA2020. The authors thank Eino Sivonen for the compactor grinding, Atte Mikkelson for carrying out the elemental analysis, Päivi Matikainen for performing MALDI-TOF MS and the direct colorimetric solid phase assay, Ritva Heinonen for the ATR-FTIR measurements and Mirja Nygård for the TGA measurements.

\section{Notes and references}

1 United States Department of Agriculture (USDA), Foreign Agricultural Service, https://apps.fas.usda.gov/psdonline/ circulars/livestock_poultry.pdf, (accessed October, 2018).

2 N. Reddy, Waste Manag., 2015, 45, 91-107.

3 M. Feughelman, J. Appl. Polym. Sci., 2002, 83, 489-507.

4 P. Schrooyen, P. Dijkstra, R. Oberthü, A. Bantjes and J. Feijen, J. Agric. Food Chem., 2000, 48, 4326-4334.

5 Y. Zhang, W. Zhao and R. Yang, ACS Sustain. Chem. Eng., 2015, 3, 2036-2042.

6 A. Poole, J. Church and M. Huson, Biomacromolecules, 2009, 10, 1-8.

7 T. Welton, Chem. Rev., 1999, 99, 2071-2083.

8 Y. Dai, J. Van Spronsen, G. Witkamp, R. Verpoorte and Y. Choi, J. Nat. Prod., 2013, 76, 2162-2173.

9 R. Swatloski, S. Spear, J. Holbrey and R. Rogers, J. Am. Chem. Soc., 2002, 124, 4974-4975.

10 H. Xie, S. Li and S. Zhang, Green Chem., 2005, 7, 606-608.

11 A. Idris, R. Vijayaraghavan, U. Rana, A. Patti and D. MacFarlane, Green Chem., 2014, 16, 2857.

12 A. Idris, R. Vijayaraghavan, U. Rana, D. Fredericks, A. Patti and D. MacFarlane, Green Chem., 2013, 15, 525-534.

13 P. Sun, Z. Liu and Z. Liu, J. Hazard. Mater., 2009, 170, 786790.

14 A. Idris, R. Vijayaraghavan, A. Patti and D. Macfarlane, ACS Sustain. Chem. Eng., 2014, 2, 1888-1894.

15 Y. Wang and X. Cao, Process Biochem., 2012, 47, 896-899. 16 Y. Ji, J. Chen, J. Lv, Z. Li, L. Xing and S. Ding, Sep. Purif. Technol., 2014, 132, 577-583.

17 B. Ma, Q. Sun, J. Yang, J. Wizi, X. Hou and Y. Yang, Environ. Sci. Pollut. Res., 2017, 24, 17711-17718.

18 A. Abbott, D. Boothby, G. Capper, L. Davies and R. Rasheed, J. Am. Chem. Soc., 2004, 126, 9142-9147.

19 E. Smith, A. Abbott and K. Ryder, Chem. Rev., 2014, 114, 11060-11082. 
20 R. Wahlström, K. Rommi, P. Willberg-Keyriläinen, D. ErciliCura, U. Holopainen-Mantila, J. Hiltunen, O. Mäkinen, H. Nygren, A. Mikkelson and L. Kuutti, ChemistrySelect, 2017, 2, 9355-9363.

21 A. Abbott, G. Capper, D. Davies, R. Rasheed and V. Tambyrajah, Chem. Commun., 2003, 1, 70-71.

22 K. Moore, D. Mangos, A. Slattery, C. Raston and R. Boulos, RSC Adv., 2016, 6, 20095-20101.

23 Z. Jiang, J. Yuan, P. Wang, X. Fan, J. Xu, Q. Wang and L. Zhang, Int. J. Biol. Macromol., 2018, 119, 423-430.

24 D. Wang and R. Tang, Mater. Lett., 2018, 231, 217-220.

25 A. Shukla, Industrial Enzymology, Discovery Publishing House Pvt Ltd, Delhi, 2009.

26 Thermo Scientific, Compat-Able ${ }^{T M}$ Protein Assay Preparation Reagent Kit, https://tools.lifetechnologies.com/content/sfs/ manuals/MAN0011434_CompatAble_Protein_Asy_Prep_Reag_UG.pdf, (accessed September 2018).

27 Thermo Scientific, Pierce ${ }^{T M}$ BCA Protein Assay Kit, https:// www.thermofisher.com/document-connect/documentconnect.html?url=https://assets.thermofisher.com/TFSAssets/LSG/manuals/MAN0011430_Pierce_BCA_ Protein_Asy_UG.pdf, (accessed September 2018).

28 K. Chan and B. Wasserman, Cereal Chem., 1993, 70, 22-26.

29 T. Thannhauser, Y. Konishi and H. Scheraga, Methods Enzymol., 1987, 143, 115-119.

30 M. Tsuboi, F. Kaneuchi, T. Ikeda and K. Akahane, Can. J. Chem., 1991, 69, 1752-1757.

31 L. Rintoul, E. Carter, S. Stewart and P. Fredericks, Biopolymers, 2000, 57, 19-28.

32 A. Barth, Biochim. Biophys. Acta, Bioenerg., 2007, 1767, 10731101.

33 T. Miyazawa and E. Blout, J. Am. Chem. Soc., 1961, 83, 712719.

34 M. Duer, N. McDougal and R. Murray, Phys. Chem. Chem. Phys., 2003, 5, 2894-2899.

35 J. Cao and C. Billows, Polym. Int., 1999, 48, 1027-1033.

36 J. Li, G. Zhang and J. Wang, Sol. Energy, 1991, 47, 443-445.

37 C. Navarro, C. Sierra and C. Ochoa-Puentes, RSC Adv., 2016, 6, 65355-65365.
38 A. Ghosh, S. Clerens, S. Deb-Choudhury and J. Dyer, Polym. Degrad. Stab., 2014, 108, 108-115.

39 D. Wang, X. Yang, R. Tang and F. Yao, Polymers, 2018, 10, 993.

40 S. Sharma, A. Gupta, S. M. S. Chik, C. G. Kee, B. M. Mistry, D. H. Kim and G. Sharma, Int. J. Biol. Macromol., 2017, 104, 189-196.

41 X. C. Yin, F. Y. Li, Y. F. He, Y. Wang and R. M. Wang, Biomater. Sci., 2013, 1, 528-536.

42 B. Ma, X. Qiao, X. Hou and Y. Yang, Int. J. Biol. Macromol., 2016, 89, 614-621.

43 X. Yin, F. Li, Y. He, Y. Wang and R. Wang, Biomater. Sci., 2013, 1, 528-536.

44 A. Poole, R. Lyons and J. Church, J. Polym. Environ., 2011, 19, 995-1004.

45 B. Bennion and V. Daggett, Proc. Natl. Acad. Sci. U. S. A., 2003, 100, 5142-5147.

46 J. Parnica and M. Antalik, J. Mol. Liq., 2014, 197, 23-26.

47 H. Monhemi, M. Housaindokht, A. Moosavi-Movahedi and M. Bozorgmehr, Phys. Chem. Chem. Phys., 2014, 16, 1488214893.

48 N. Sun, M. Rahman, Y. Qin, M. Maxim, H. Rodríguez and R. Rogers, Green Chem., 2009, 11, 646-655.

49 Y. Zhang, R. Yang and W. Zhao, J. Agric. Food Chem., 2014, 62, 2745-2751.

50 W. Zhao, R. Yang, Y. Zhang and L. Wu, Green Chem., 2012, 14, 3352-3360.

51 A. Woodin, Biochem. J., 1954, 57, 99-109.

52 S. Jayasundera and W. Schmidt, Microcrystalline Avian Keratin Protein Fibers, in Natural Fibers, Plastics and Composites, ed. F. Wallenberger and N. Weston, Kluwer Academic Publishers, Boston, 2004, vol. 4, pp. 51-65.

53 J. Cao, Thermochim. Acta, 1999, 335, 5-9.

54 M. Zoccola, A. Aluigi and C. Tonin, J. Mol. Struct., 2009, 938, 35-40.

55 N. Reddy and Y. Yang, J. Polym. Environ., 2007, 15, 81-87.

56 D. Rao and V. Gupta, J. Appl. Polym. Sci., 1992, 46, 1109-1112. 